\title{
A nonrandom association of sarcoidosis in patients with gastrointestinal stromal tumor and other sarcomas
}

Rare Tumors

Volume 10: 1-7

(C) The Author(s) 2018

Reprints and permissions: sagepub.co.uk/journalsPermissions.nav DOI: $10.1177 / 2036361318787626$ journals.sagepub.com/home/rtu (SSAGE

\author{
Andrea P Espejo', Jeremy L Ramdial'2, Breelyn A Wilky ${ }^{2,3}$, \\ Darcy A Kerr ${ }^{4}$ and Jonathan C Trent ${ }^{2,3}$
}

\begin{abstract}
In patients with sarcoma, concomitant malignancy is found in $1.2 \%-2.5 \%$ of cases. Previous studies have demonstrated conflicting results in terms of positive or negative effects on cancer prognosis with comorbid sarcoidosis. Additionally, there are no data determining whether an association between sarcoidosis and sarcomas exists. Finding an association between the two entities could prevent inadvertent upstaging of a primary sarcoma based on pulmonary nodularity mistaken for metastatic disease. Here, we will describe eight sarcoma patients with concomitant occurrence of sarcoidosis identified since 2007. Eight patients with diagnosis of both sarcoma and sarcoidosis were identified over the period of $2007-2016$ at a single sarcoma center. Clinical and historical data including presentation, histology, treatment, and outcome was tabulated for analysis. The standardized incidence ratio was calculated for the state of Florida and our hospital catchment area. We compared the observed incidence to the expected incidence if the two entities were to be unrelated. Sarcoma subtype was gastrointestinal stromal tumor in five patients, the remaining three cases were unclassified spindled and epithelioid cell sarcoma, uterine leiomyosarcoma, and myxofibrosarcoma. Sarcoidosis was diagnosed before sarcoma in three patients, after sarcoma in four patients, and at the same time of sarcoma diagnosis in one patient. From our series, three patients have shown no progression of sarcoma, two are alive with sarcoma, two died due to progression of sarcoma, and one was lost to follow up. Statistical analyses showed a standardized incidence ratio of 305 (95\% confidence interval: |3| -556) for the state of Florida and standardized incidence ratio of $950(95 \% \mathrm{Cl}: 407$ - 1727) for our catchment area. This case series points to a statistically robust, nonrandom association between sarcoma and sarcoidosis that has not been previously described. Presumed metastatic sarcoma should be considered for biopsy particularly with demographic characteristics or imaging features suggestive of sarcoidosis.
\end{abstract}

\section{Keywords}

Sarcoma, sarcoidosis, soft tissue sarcoma, metastatic sarcoma, gastrointestinal stromal tumor

Date received: 12 April 20I8; accepted: 15 June 2018

\footnotetext{
'Jackson Health System, Department of Internal Medicine, University of Miami Miller School of Medicine, Miami, FL, USA

2Department of Hematology/Oncology, Sylvester Comprehensive Cancer Center, University of Miami Miller School of Medicine, Miami, FL, USA

${ }^{3}$ Sarcoma Research Program, Sylvester Comprehensive Cancer Center, University of Miami Miller School of Medicine, Miami, FL, USA
}

\author{
${ }^{4}$ Department of Pathology, Sylvester Comprehensive Cancer Center, \\ University of Miami Miller School of Medicine, Miami, FL, USA \\ Corresponding author: \\ Andrea P Espejo, Jackson Health System, Department of Internal \\ Medicine, University of Miami Miller School of Medicine, 16II NW 12th \\ Avenue, Miami, FL 33136, USA. \\ Email: andrea.espejofreire@jhsmiami.org
}




\section{Introduction}

Sarcomas are a rare group of malignant tumors that arise from mesenchymal tissues. They compromise less than $1 \%$ of all cancers in adults with 15,600 new cases diagnosed in the United States in 2016. ${ }^{1}$ Histopathology of sarcomas is highly heterogeneous with over 200 distinct histologic subtypes described. ${ }^{1,2}$ The most prevalent subtype is gastrointestinal stromal tumor (GIST) followed by fibrosarcoma, liposarcoma, leiomyosarcoma, synovial sarcoma, and malignant peripheral nerve sheath tumor (MPNST). ${ }^{2,3}$ Most sarcomas are sporadic; however, there are key environmental causative factors including history of ionizing radiation, exposure to vinyl chloride, and human herpes virus 8 (HHV-8). ${ }^{4}$ Known genetic syndromes associated with a predisposition to sarcoma include Li-Fraumeni syndrome, retinoblastoma, neurofibromatosis type 1, Carney's triad, and familial adenomatous polyposis syndrome. In addition, the association of GIST with multiple other malignancies and desmoid tumor is well documented. .5

On the contrary, sarcoidosis is a multisystem disease of unknown etiology. The incidence of sarcoidosis has been estimated at approximately 1-35 cases per 100,000 with the highest rates among Northern Europe and African American populations. ${ }^{7}$ Any organ may be involved, but the most common presenting sites are the lung and lymphatic system. ${ }^{8}$ It is thought that sarcoidosis occurs after antigen exposure induces recruitment, activation, and aggregation of macrophages with granulomatous inflammation. Subsequently, the disease follows one of two pathways: resolution or progression leading to fibrosis. The susceptibility and spectrum of manifestations is driven by genetic susceptibility; strong association has been found for MHC-2 alleles HLA DRB $1 * 3$ for remitting disease and HLA DRB $1 * 14$ and 15 for a chronic course. Co-stimulator molecules and cytokine polymorphisms have also been linked with different clinical manifestations. ${ }^{7,9}$

Several case reports and descriptive studies have suggested an increased risk of malignancy in patients with sarcoidosis. Concomitant occurrence of cancer is found in $1.2 \%-2.5 \%$ of patients with sarcoidosis. ${ }^{10}$ In 2015 , a metaanalysis of 16 studies (10 cohorts and 6 case-control studies) involving a total of 27,268 patients and 1952 cancer cases reported a relative risk (RR) of 1.19 (confidence interval (CI): 1.07-1.32) for the development of any invasive cancer. The association was stronger for skin (RR: 2.00) and hematologic malignancies (RR: 1.92) during the first 10 years after diagnosis. ${ }^{11}$ The implications of sarcoidosis development following the diagnosis of malignancy are also reported. A descriptive study found 64 patients (from 1980 to 2011) with a cancer diagnosis who subsequently developed sarcoidosis. In this cohort, the associated malignancies were breast, lymphoma, lung, and testicular cancer. Results showed that among patients developing sarcoidosis, 53\% developed recurrence of the primary cancer and $67 \%$ developed a secondary malignancy. These findings showed that sarcoidosis is often discovered before or at diagnosis of cancer. Still, it is associated with recurrence or second malignancy in a significant proportion of patients. ${ }^{12}$

The association of sarcoma and sarcoidosis is quite rare in the literature; there are no published series, and the implications of this coexistence are not known. We found single patient case reports (six since 1978) describing an association of sarcoidosis with two cases of osteosarcoma and one case each of GIST, gastric leiomyosarcoma, angiosarcoma, and one reported as low-grade sarcoma. ${ }^{13-18}$ Herein, we describe the concomitant occurrence of sarcoidosis in eight patients diagnosed with sarcoma at a comprehensive sarcoma center since 2007. This case series constitutes the largest of its kind, suggests a nonrandom association of these two conditions, and will directly impact clinical practice by raising awareness of a potential mimic of metastasis.

\section{Materials and methods}

\section{Patients and pathology tissues}

This case series includes eight patients with concomitant soft-tissue sarcoma and sarcoidosis. After initial protocol review by University of Miami Institutional Review Board, approval to proceed was given and further review waved according to the size and retrospective nature of the study. The data supporting the findings of this study are available from the corresponding author, A.P.E., on request.

Clinical characteristics and pathology data were retrieved from patient records at The University of Miami, Sylvester Comprehensive Cancer Center. Five of the eight tissue samples were available and evaluated by an experienced sarcoma pathologist who confirmed sarcoidosis and sarcoma histology. Unavailable samples correspond to the cases were sarcoidosis was diagnosed several years prior to sarcoma. Demographic and clinical information including presentation, treatment, histology, and outcome-related variables were tabulated for analysis.

\section{Statistical analyses}

The standardized incidence ratio (SIR) was used to determine whether the association between sarcoma and sarcoidosis was due to chance. This ratio is calculated by the number of events observed divided by the number of events expected. The overall population used was the hospital catchment area (6.5 million people) and the state of Florida (20.2 million people). SIR has shown to be a useful tool to analyze incidence data prior to large, expensive, population-based studies. ${ }^{19}$ We calculated $95 \%$ CIs of SIR using the Vandenbroucke ${ }^{20}$ method. Descriptive statistics were used to analyze demographic, tumor, and treatment data. 


\section{Results}

\section{Clinical characteristics}

Eight patients with diagnosis of soft-tissue sarcoma and sarcoidosis seen between 2007 and 2016 were analyzed in this case series. Table 1 shows the clinical characteristics of these patients, which includes six females $(75 \%)$ and two males $(25 \%)$. Ethnicity was Caucasian in five patients, two African American, and one Hispanic. Pain was the most common presenting symptom; three cases presented as painless mass, two as abdominal pain, two cases as incidental finding, there was no data on presentation for one patient.

\section{Sarcoma histology}

Sarcoma subtypes included GIST in five patients with one patient also diagnosed with concurrent breast cancer and a metanephric adenoma. Mutation analysis of GIST cases revealed mutation of KIT exon 11 in two patients, PDGFR exon 12 in one patient, PDGFR exon 18 in one patient, and one patient had no mutational analysis done. Another case was an unclassified spindled and epithelioid cell sarcoma that coexisted with tenosynovial giant cell tumor. Finally, there was one patient with uterine leiomyosarcoma, and one diagnosed with myxofibrosarcoma. Two patients were diagnosed at stage I, two at stage II, two at stage III, and two at stage IV.

\section{Patient presentations}

In three patients, sarcoidosis was diagnosed before the onset sarcoma, four patients were diagnosed with sarcoidosis after the sarcoma diagnosis, and one patient was diagnosed with sarcoidosis at presentation of sarcoma with coexistent metastatic disease and sarcoidosis in the lungs. When sarcoidosis was diagnosed after sarcoma, the average time for diagnosis was 16 months (range: 7 months to 2.5 years). Sarcoidosis was diagnosed after abnormal chest imaging for surveillance in three patients. In one patient, sarcoidosis was found incidentally after undergoing imaging for suspected pulmonary embolism, and in one patient sarcoidosis was found by pathology in all abdominal lymph nodes and the spleen resected at surgery for recurrence. Data regarding presentation were unavailable in the three patients with history of sarcoidosis prior to the diagnosis of cancer. Seven cases were of pulmonary sarcoidosis and one was found in abdominal lymph nodes and spleen. Figure 1 shows the imaging and pathology findings of patient number 2. The surveillance positron emission tomography/computed tomography (PET/CT) scan was initially thought to represent metastasis, but subsequent biopsy revealed the diagnosis of sarcoidosis.

\section{Treatment and outcomes}

All GIST cases were treated with imatinib and two of them were subsequently treated with sunitinib. The patient with unclassified spindled and epithelioid cell sarcoma that coexisted with tenosynovial giant cell tumor was treated with resection and local radiation. The patient with uterine leiomyosarcoma was treated with surgical resection plus multiple lines of chemotherapy including gemcitabine/docetaxel, doxorubicin, ifosfamide, and palliative radiation. Finally, the case of myxofibrosarcoma was given doxorubicin plus cyclophosphamide followed by resection and local radiation.

With a median follow-up period of 29.5 months, three of our sarcoma cases have not shown progression, two patients are alive with current evidence of sarcoma, two patients died due to complications from the primary tumor, and one patient was lost to follow-up. Four out of five patients with GIST are alive on follow-up, two with no evidence of disease and two alive with disease, the remaining case was lost to follow-up. Regarding non-GIST STS, 2 patients are deceased, and one is alive with no evidence of disease.

\section{Statistical analysis}

The annual incidence of sarcoidosis is estimated at 1-35 cases per $100,000^{7}$ and the incidence of soft-tissue sarcoma is 3.7 cases per 100,000 in the general US population. ${ }^{1} \mathrm{By}$ December 2016, the catchment area of our hospital was 6.5 million individuals and the population of the state of Florida 20.2 million. Assuming independence, the expected number of patients with both sarcoma and sarcoidosis is 2.6 for the state of Florida and 0.84 for our catchment area in the period of 2007-2016, corresponding to the time interval when our cases were initially seen. Assuming all sarcoma patients in the state of Florida were to be seen at our center, the estimated risk of having sarcoma and sarcoidosis was significantly higher than expected $(\mathrm{SIR}=305 ; 95 \% \mathrm{CI}$ : 131-556), indicating the probability of coexistence of these two entities is $205 \%$ higher than expected. Similarly, the estimated risk of both diseases was even greater if one were to consider our catchment area of five counties in South Florida (SIR $=950 ; 95 \%$ CI: 407-1727), indicating an incidence $850 \%$ higher than expected.

\section{Discussion}

The objective of this retrospective case series was to determine whether there was evidence of an association between soft-tissue sarcoma and sarcoidosis or if this observation is due to chance alone. Sarcoidosis has been previously associated with a twofold increased risk of development of cancer, most commonly lung, cutaneous, and hematologic malignancies. ${ }^{11,22}$ Some hypothesize the finding of sarcoidosis following cancer diagnosis may represent an 


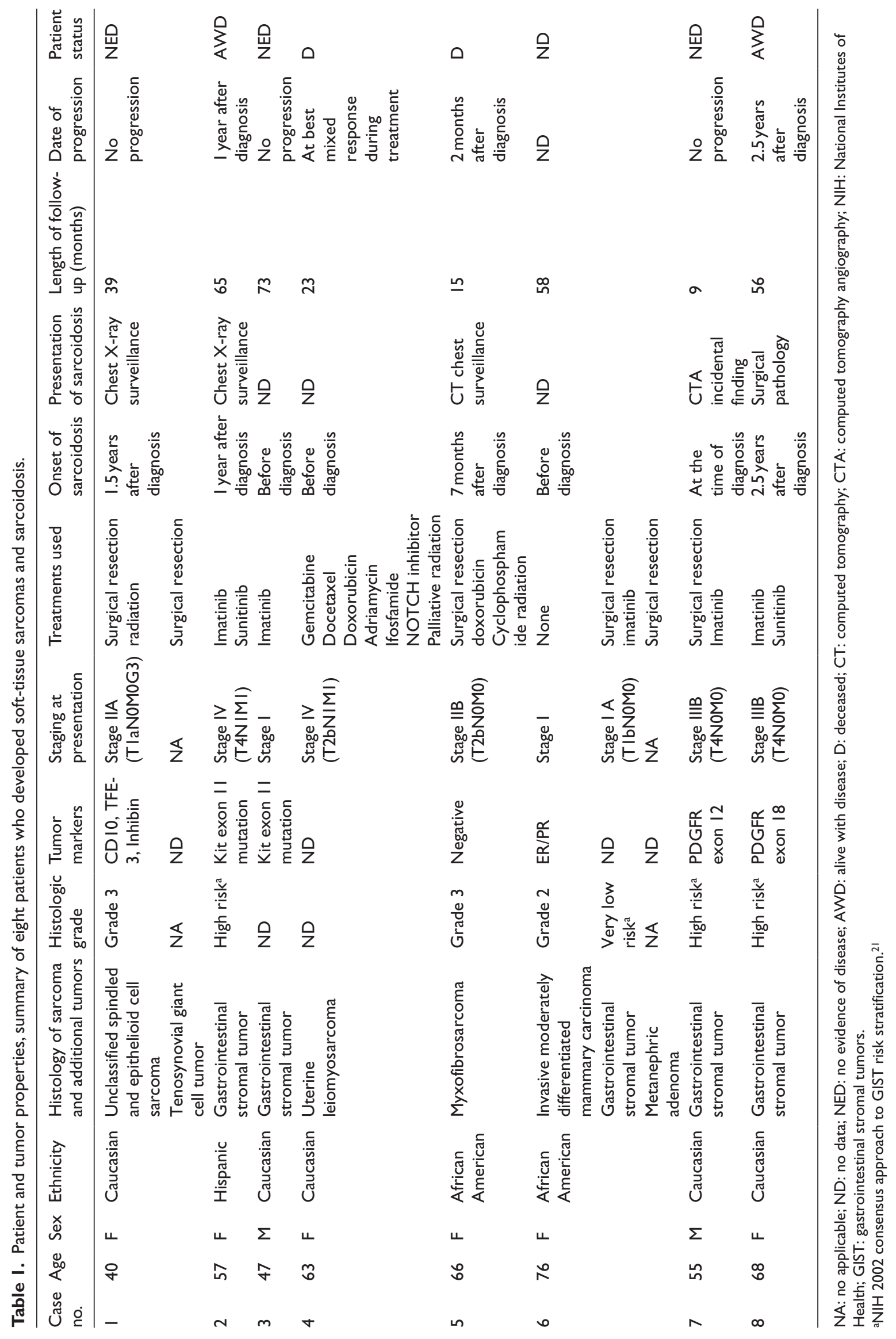




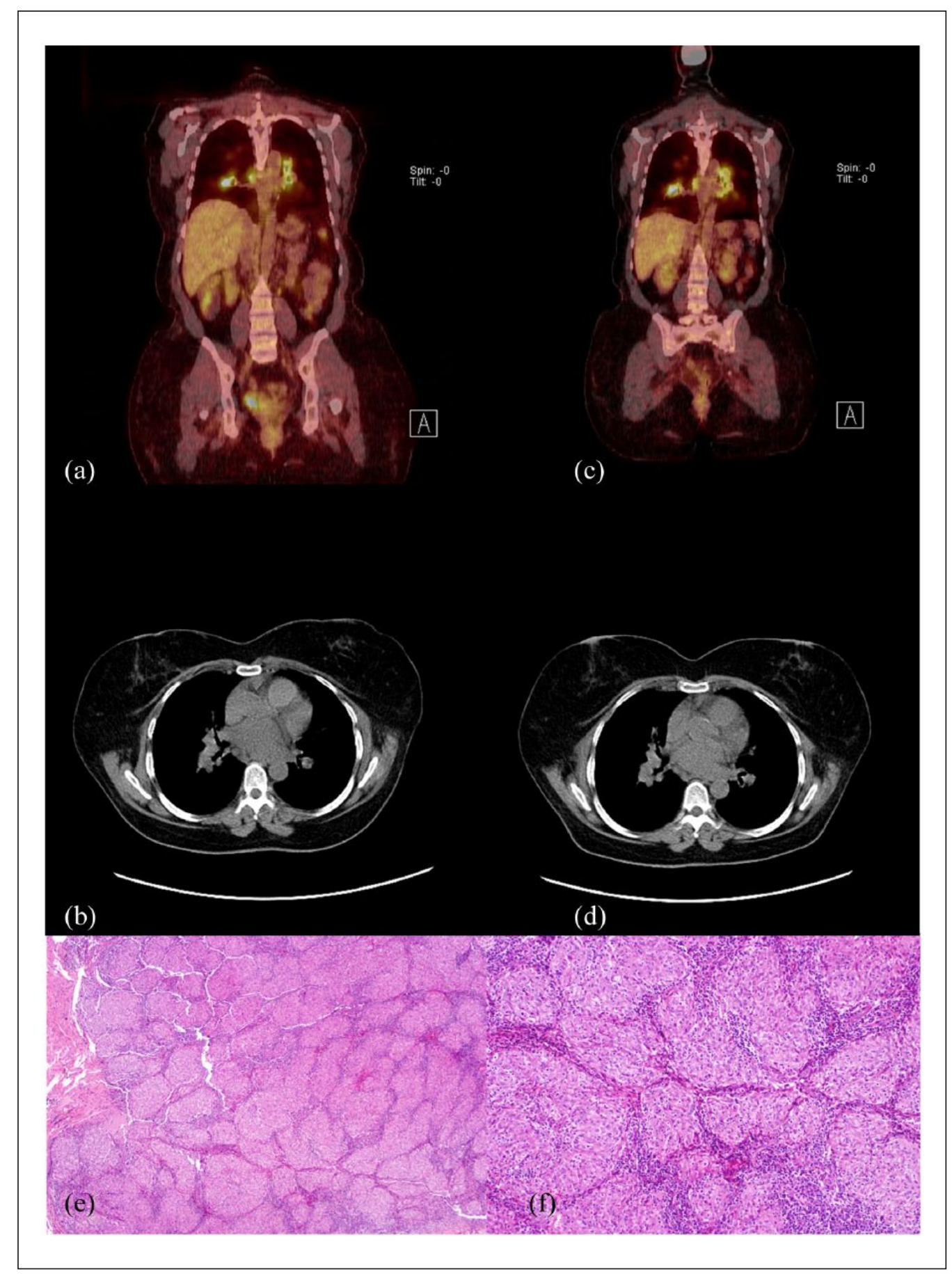

Figure I. PET/CT and pathology slides of case no. 2. (a, b) Initial PET/CT and chest CT showing para-hilar lymph nodes involvement. (c, d) PET/CT and chest CT at 6 months showing stability of the lymph nodes. Patient subsequently underwent endobronchial biopsy showing non-caseating granulomas consistent with sarcoidosis. (e) Lymph node diffusely replaced by compact non-necrotizing granulomas (4x). (f) Granulomas are formed of clusters of epithelioid histiocytes and have well demarcated margins $(10 x)$.

immunologic response to the underlying tumor. This "sarcoid-like reaction" predominantly occurs in patients with Hodgkin disease and, to a lesser extent, in individuals with other hematologic malignancies and solid tumors. ${ }^{23}$ Our results represent the first series of patients with soft-tissue sarcoma and sarcoidosis and point to a novel, nonrandom, statistically robust association between these two entities. Moreover, our results likely underestimate the association as we are likely missing patients with occult sarcoidosis not detected on routine imaging as well as the fact our center 
does not see all sarcoma patients in our state nor in our catchment area.

Prior reports in other types of cancer have been inconsistent in terms of prognostic implications. In patients with Hodgkin lymphoma and gastric carcinoma, the presence of sarcoid reactions has been associated with favorable prognosis. ${ }^{22,23}$ On the contrary, as previously mentioned, a large retrospective cohort of sarcoidosis following the diagnosis of cancer showed that "sarcoid reactions" were associated with recurrence of disease in 53\% of cases and secondary malignancies in $67 \%$. The authors concluded that finding sarcoidosis in cancer patient should mandate closer cancer surveillance. ${ }^{12}$ The cases we presented have mixed outcomes from their sarcoma. Three of the patients have no evidence of disease, two with sarcoidosis discovered after diagnosis of cancer (follow-up 36 and 4 months, respectively) and one with history of sarcoidosis before diagnosis of sarcoma (follow-up of 8.8 years). In three of our cases, the presence of sarcoidosis coexisted with sarcoma progression.

Interestingly, five out of our eight patients have GIST which is significantly higher than expected for the relative incidence of GIST to other STS (1:6). A possible explanation is the role of colony-stimulating factor 1 (CSF-1) in the pathogenesis of both malignancy and sarcoidosis. CSF-1, and its receptor CSF-1R, is overexpressed and drives proliferation of many malignancies and tumor-associated macrophages (TAM) which are involved in tumor progression. In patients with sarcoidosis, CSF-1, CSF-1R, and interleukin (IL)-3 (another ligand of CSF-1R) are increased in bronchoalveolar lavage samples and decreases after treatment with steroids. CSF-1R is a receptor tyrosine kinase (RTK) and shares a significant proportion of amino acid identity with KIT, PGFR, and FLT3. This family of RTKs is an essential component of signaling transduction in cellular growth, differentiation, and motility. ${ }^{24}$ In the case of GIST, there is also evidence that CSF-1R mutation (D802V) results in alteration at the binding site leading to resistance to imatinib. ${ }^{25}$ These findings illustrate the possibility of an immune reaction in sarcoma-related sarcoidosis that may be driven by oncogenic tumor pathways. For this reason, additional investigation into the molecular mechanism of sarcoidosis including the possibility of an immune response to occult metastatic sarcoma could provide better understanding of the disease and open new therapeutic approaches.

Clinically, our findings are particularly important as the lung is the most common site of sarcoma metastasis. Thus, not all patients with lung nodules are subjected to a biopsy to confirm metastatic sarcoma. Conversely, the results of this study support the idea that patients with presumed metastatic sarcoma should be considered for a biopsy to exclude other entities such as sarcoidosis. This could be critical as a patient with erroneously presumed metastatic sarcoma may not be treated with curative intent. On the contrary, we agree with previous recommendations stating that the presence of sarcoidosis dictates closer monitoring as it is not always an isolated benign finding. When sarcoidosis is discovered as part of surveillance, biopsy is not enough to secure that sarcoidosis is an isolated process as often not all suspicious sites are sampled. Noninvasive imaging techniques are under investigation to address this problem. Fluoride-18-methyltyrosine (18F-FMT) has been shown to have uptake by malignancy (lymphoma, brain tumors, and experimental tumor models) but not by sarcoidosis. A recent study of 24 patients with sarcoidosis and 17 patients with lung cancer found lung cancer lesions showed uptake of both 18F-FDG and 18F-FMT tracers, while patients with sarcoidosis had uptake of only $18 \mathrm{~F}$ FDG. Introduction of such techniques may be very important for better surveillance of cancer patients. ${ }^{26}$

\section{Conclusion}

Although our results should be viewed as exploratory due to the small sample size, this series of eight sarcoma patients with sarcoidosis indicates an intriguing association which may be much more common than previously thought. This association may have impact on identification of patients falsely diagnosed with metastatic disease. Moreover, the association opens the door to additional studies to fully understand the molecular pathogenesis. Our observations highlight the importance of future investigation into sarcoma-related sarcoidosis.

\section{Acknowledgements}

The authors thank Dr Leonardo Tamariz, for his assistance in the statistics review of this research. J.C.T. and B.A.W. identified patients from their clinical rosters and conceived the study. A.P.E. and J.L.R. researched the literature and designed the study. A.P.E., J.L.R., and J.C.T. were involved in protocol development and institutional review board (IRB) approval. A.P.E. and J.L.R. gathered patient data by chart review. D.A.K. reviewed pathology slides to confirm the diagnosis of sarcoma and sarcoidosis histology. A.P.E. wrote the first draft of the manuscript. All authors reviewed and edited the manuscript and approved the final version of the manuscript.

\section{Conflict of interest}

The author(s) declared no potential conflicts of interest with respect to the research, authorship, and/or publication of this article.

\section{Ethical approval}

University of Miami Institutional Review Board granted approval and waived further review after initial protocol submission.

\section{Funding}

The author(s) received no financial support for the research, authorship, and/or publication of this article.

\section{Informed consent}

Informed consent was waived by the University of Miami Institutional Review Board. 


\section{Previous presentations/publications}

Abstract submitted for ASCO 2017 annual meeting and accepted for publication at abstracts.asco.org.

\section{References}

1. Siegel RL, Miller KD and Jemal A. Cancer statistics, 2016. CA Cancer J Clin 2016; 66(1): 7-30.

2. Jo VY and Doyle LA. Refinements in sarcoma classification in the current 2013 World Health Organization classification of tumours of soft tissue and bone. Surg Oncol Clin N Am 2016; 25(4): 621-643.

3. Coindre JM, Terrier P, Guillou L, et al. Predictive value of grade for metastasis development in the main histologic types of adult soft tissue sarcomas: a study of 1240 patients from the French Federation of Cancer Centers sarcoma group. Cancer 2001; 91(10): 1914-1926.

4. Hui JY. Epidemiology and etiology of sarcomas. Surg Clin North Am 2016; 96(5): 901-914.

5. Pandurengan RK, Dumont AG, Araujo DM, et al. Survival of patients with multiple primary malignancies: a study of 783 patients with gastrointestinal stromal tumor. Ann Oncol 2010; 21(10): 2107-2111.

6. Dumont AG, Rink L, Godwin AK, et al. A nonrandom association of gastrointestinal stromal tumor (GIST) and desmoid tumor (deep fibromatosis): case series of 28 patients. Ann Oncol 2012; 23(5): 1335-1340.

7. Valeyre D, Prasse A, Nunes H, et al. Sarcoidosis. Lancet 2014; 383(9923): 1155-1167.

8. Heinle R and Chang C. Diagnostic criteria for sarcoidosis. Autoimmun Rev 2014; 13(4-5): 383-387.

9. Baughman RP, Lower EE and Du Bois RM. Sarcoidosis. Lancet 2003; 361(9363): 1111-1118.

10. Reich JM. Neoplasia in the etiology of sarcoidosis. Eur $J$ Intern Med 2006; 17(2): 81-87.

11. Bonifazi M, Bravi F, Gasparini S, et al. Sarcoidosis and cancer risk: systematic review and meta-analysis of observational studies. Chest 2015; 147(3): 778-791.

12. Kiess A, Wang H, Travis W, et al. Sarcoid in cancer patients: clinical characteristics and associated disease status. Sarcoidosis Vasc Diffuse Lung Dis 2015; 32(3): 200-207.

13. Akagawa S, Hashimoto K, Murota N, et al. A case of sarcoidosis associated with gastric leiomyosarcoma. Nihon Kyobu Shikkan Gakkai Zasshi 1987; 25(8): 896-900.
14. Badmaeva VV. Association of angiosarcoma with sarcoidosis of the lung. Arkh Patol 1970; 32(5): 67-69.

15. Sybert A and Butler TP. Sarcoidosis following adjuvant highdose methotrexate therapy for osteosarcoma. Arch Intern Med 1978; 138(3): 488-489.

16. Vital C, Bonnin A, Dumas P, et al. Acute sarcoid neuropathy developed 4 months after the occurrence of a gastrointestinal stromal tumor (GIST). Sarcoidosis Vasc Diffuse Lung Dis 2009; 26(1): 69-72.

17. Okada K, Sasaki M, Nanjo H, et al. Benign lesions in cancer patients: case 2. Unusual association of osteosarcoma and sarcoidosis. J Clin Oncol 2005; 23(3): 641-643.

18. Majori M, Anghinolfi M, Gnetti L, et al. Pulmonary metastases from low grade sarcoma in a patient with pulmonary sarcoidosis. Sarcoidosis or sarcoid-like Reaction? Sarcoidosis Vasc Diffuse Lung Dis 2016; 33(2): 171-174.

19. Boyle P and Parkin D. Cancer registration: principles and methods. Statistical methods for registries. IARC Sci Publ 1991; (95): 126-158.

20. Vandenbroucke JP. A shortcut method for calculating the 95 per cent confidence interval of the standardized mortality ratio. Am J Epidemiol 1982; 115(2): 303-304.

21. Fletcher C, Berman J, Corless C, et al. Diagnosis of gastrointestinal stromal tumors: a consensus approach. Hum Pathol 2002; 33(5): 459-465.

22. Chowdhury FU, Sheerin F, Bradley KM, et al. Sarcoid-like reaction to malignancy on whole-body integrated $18 \mathrm{~F}-\mathrm{FDG}$ PET/CT: prevalence and disease pattern. Clin Radiol 2009; 64(7): 675-681.

23. Tanimoto $\mathrm{Y}$ and Kataoka M. Sarcoidosis and malignancy. Japanese J Chest Dis 2013; 72(8): 838-845.

24. Kumari A, Silakari O and Singh RK. Recent advances in colony stimulating factor-1 receptor/c-FMS as an emerging target for various therapeutic implications. Biomed Pharmacother 2018; 103: 662-679.

25. Da PDSFC, Beauchêne ICD, Panel N, et al. Insight on mutation-induced resistance from molecular dynamics simulations of the native and mutated CSF-1R and KIT. PLoS ONE 2016; 11(7): e0160165.

26. Kaira K, Oriuchi N, Otani Y, et al. Diagnostic usefulness of fluorine-18-alpha-methyltyrosine positron emission tomography in combination with $18 \mathrm{~F}$-fluorodeoxyglucose in sarcoidosis patients. Chest 2007; 131(4): 1019-1027. 